\title{
Prise en compte des pré-soutènements dans le dimensionnement des tunnels
}

\section{A. GUILLOUX}

S. BRETELLE

Terrasol

Immeuble Hélios

72, avenue Pasteur,

93100 Montreuil

\section{F. BIENVENUE}

ENPC,

28, rue des Saints-Pères, 75007 Paris

Les méthodes usuelles de calcul des tunnels prenant en compte les phénomènes tridimensionneis et d'interaction sol-structure ont largement évolué récemment. Toutefois. la présence de pré-soutènements, de plus en plus fréquemment utilisés notamment pour les projets en site urbain, reste encore difficile à prendre en compte dans les méthodes existantes. L'article présente les résultats d'études théoriques permettant de proposer une méthodologie de généralisation des approches "convergence-confinement $)$ aux cas de tunnels utilisant les méthodes de pré-soutènements (pré-voûtes, voûtes-parapluies, jet-grouting, etc.).

\section{Effects of pre-linings on the tunnels design}

The usual design methods of turnels, taking into consideration the tridimensional phenomena and the interactions between the structures and the ground, have been much developped recently. But the presence of a pre-linings $n$, more and more frequently used mainly for projects in urban environment, remains difficult to considers in the existing approachs. The paper describes the results of theoretical analysis, and propose a methodology generalizing the classical $\propto$ convergenceconfinement $s$ approaches for the tunnels using pre-lining methods (pre-vault, umbrellas, jet-grouting...). 


\section{Introduction}

Depuis une vingtaine d'années, il est devenu d'usage courant de prendre en considération les phénomènes tridimensionnels intervenant dans le comportement d'un ouvrage souterrain au voisinage du front de taille, en utilisant les approches du type méthode convergence-confinement.

Le principe consiste à déterminer la valeur du taux de déconfinement, directement lié à la convergence de l'excavation, à une distance donnée du front d'un tunnel dans le but de réaliser une étude bidimensionnelle au niveau de cette section, le plus souvent à l'aide d'un calcul par éléments finis, permettant de tenir compte de la géométrie exacte de l'ouvrage et du phasage de réalisation. Cette démarche se justifie par le fait que des calculs numériques tridimensionnels restent encore aujourd'hui lourds à mettre en cuvre.

Des progrès considérables ont été réalisés ces dernières années dans l'évaluation de la variation du taux de déconfinement en arrière du front d'un tunnel sou- tenu. La rigidité du soutènement peut ainsi être prise en compte dans l'évaluation des contraintes et des convergences observées loin en arrière du front dans le cas d'un tunnel simplement soutenu (Bernaud, Rousset, 1992). Toutefois, les méthodes actuelles d'évaluation du taux de déconfinement restent limitées aux cas des soutènements mis en cuvre en arrière du front.

Or, de plus en plus de projets souterrains sont à réaliser dans des terrains de qualité médiocre et dans des environnements sensibles aux déformations, ce qui conduit à utiliser fréquemment des techniques de présoutènement (pré-voûtes, voûtes-parapluies, boulonnage du front de taille), qui viennent agir sur le terrain dans la zone du front de taille et parfois même loin en avant de celui-ci. Mais les méthodes actuelles d'évaluation des taux de déconfinement ne permettent pas de prendre en compte l'effet de ces modifications de contraintes et de comportement en avant du front.

L'objet de cet article est ainsi de proposer une méthodologie et des résultats destinés à appliquer l'approche convergence-confinement dans le cadre de la mise en place de pré-soutènements.

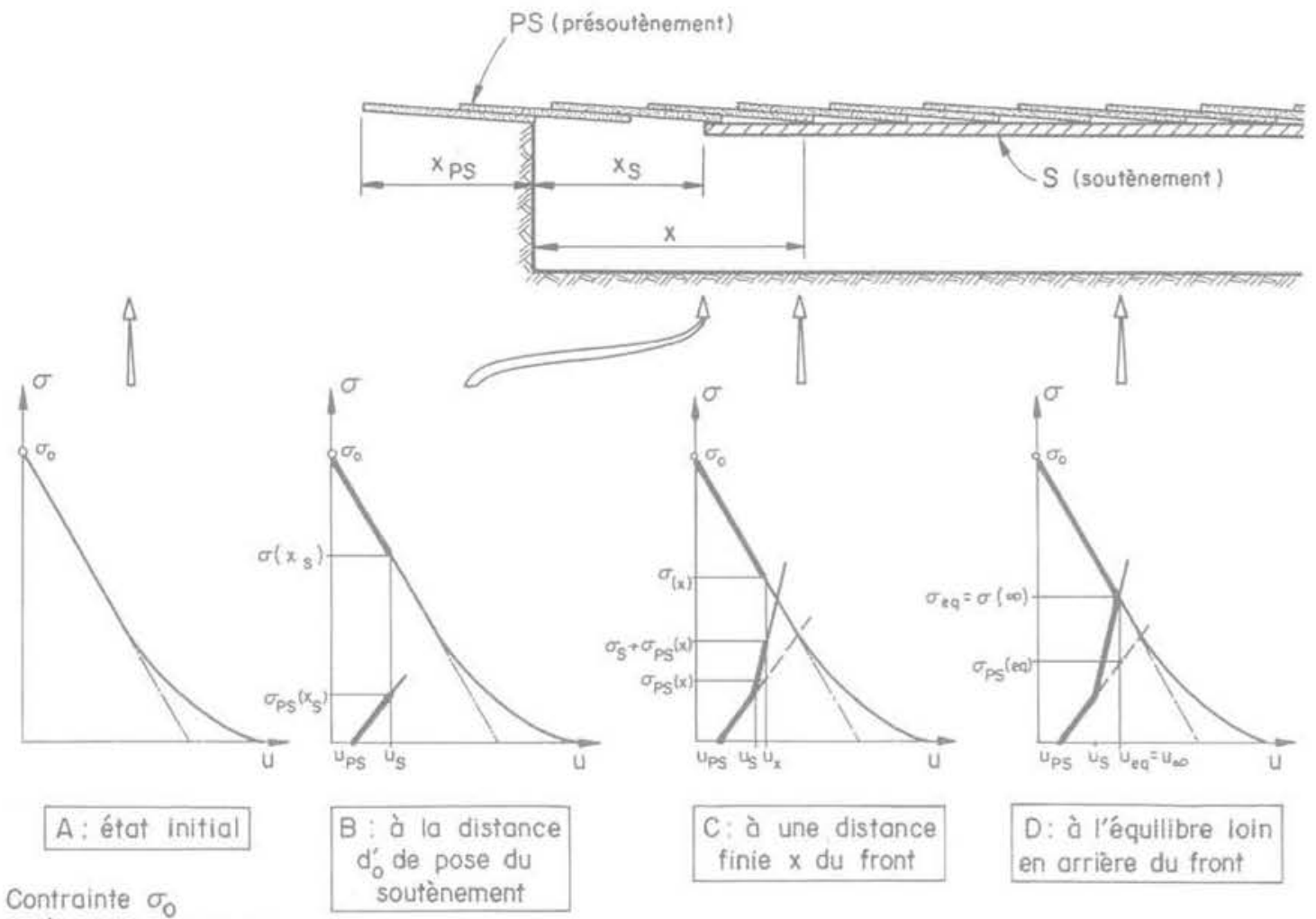

entièrement reprise par le terrain non excové

$$
\text { Contrainte } \sigma_{0} \text { reprise por }\left\{\begin{array}{l}
\text { le pré-soutènement. } \sigma_{\mathrm{PS}} \\
\text { le soutènement } \sigma_{S} \\
\text { dissipation du fait de la convergence. } \sigma_{0}-\sigma \\
\text { la présence du front (B et C }), \sigma-\left(\sigma_{S}+\sigma_{\mathrm{PS}}\right)
\end{array}\right.
$$




\section{Évolution des contraintes au cours du processus de creusement d'un tunnel}

Lorsqu'on vient de réaliser une excavation au sein d'un massif de terrain, la contrainte initiale régnant dans ce massif se trouve répartie sous trois formes au niveau d'une section située au voisinage du front de taille (Fig. 1):

- l'énergie potentielle associée à cette contrainte est partiellement dissipée par déplacement du terrain (phénomène de convergence) ;

- une part de la contrainte est reprise par la présence du terrain non excavé (influence du front), pour les sections situées à faible distance du front

- une part de la contrainte est reprise par les diverses formes de (pré)-soutènements mis en place.

Il apparait que l'influence des différents éléments permettant de reprendre cette contrainte initiale (terrain non excavé, différentes formes de (pré)-soutènements), n'est pas limitée aux zones où ces éléments sont présents. Ainsi, à un ou deux diamètres en arrière du front, l'état d'équilibre de l'excavation n'est pas encore atteint et l'influence du front se fait encore sentir. Cette remarque a conduit à l'introduction de la notion de pression fictive de soutènement destinée à tenir compte de la présence du front de taille et de son influence au niveau d'une section située à une distance $\mathrm{x}$ en arrière de celui-ci : par cet intermédiaire, l'étude d'un tunnel en cours de creusement peut alors se ramener à l'étude d'un tunnel infini soumis à une certaine pression fictive $\sigma(\mathrm{x})$, définie à partir de la contrainte initiale $\sigma_{0}$ et du taux de déconfinement $\lambda(\mathrm{x})$ :

$$
\lambda(x)=1-\frac{\sigma(x)}{\sigma_{0}}
$$

En pratique, l'étude d'une section de tunnel (pré-) soutenu situé à une distance finie du front est en général conduite de la façon suivante (Fig, 2):

- 1: détermination de la courbe de comportement du terrain (courbe de convergence du terrain) ;

-2 : détermination de la courbe donnant la convergence $\mathrm{u}(\mathrm{x})$ en fonction de la distance $\mathrm{x}$ par rapport au front (en avant ou en arrière de celui-ci) :

-3 : estimation des différentes formes de répartition de la contrainte initiale et en particulier de la valeur de la pression fictive correspondant à la part de la contrainte $\sigma_{0}$ reprise par la présence du front;

- 4: calcul numérique à l'aide d'une modélisation bidimensionnelle de la section considérée dans lequel on appliquera sur la surface d'excavation la pression fictive dèterminée précédemment.

Le point le plus délicat d'une telle approche demeure l'étape 2 de détermination de la courbe de convergence en fonction de la distance au front. Cette approche se fait en général en considérant un problème axisymétrique et, selon les cas, en prenant en compte les raideurs et les distances de pose des divers (pré-)soutènements ; en fonction des distances de pose des (pré-)soutènements $\left(\mathrm{x}_{\mathrm{ns}}\right.$ et) $\mathrm{x}_{\mathrm{s}}$ et de la distance $\mathrm{x}$ de la section considérée, on détermine la convergence $\mathrm{u}(\mathrm{x})$ au niveau de la section :

- le problème a été initialement ramené au cas simple du tunnel non soutenu; c'est celui présenté dans les recommandations de l'AFTES (1984);

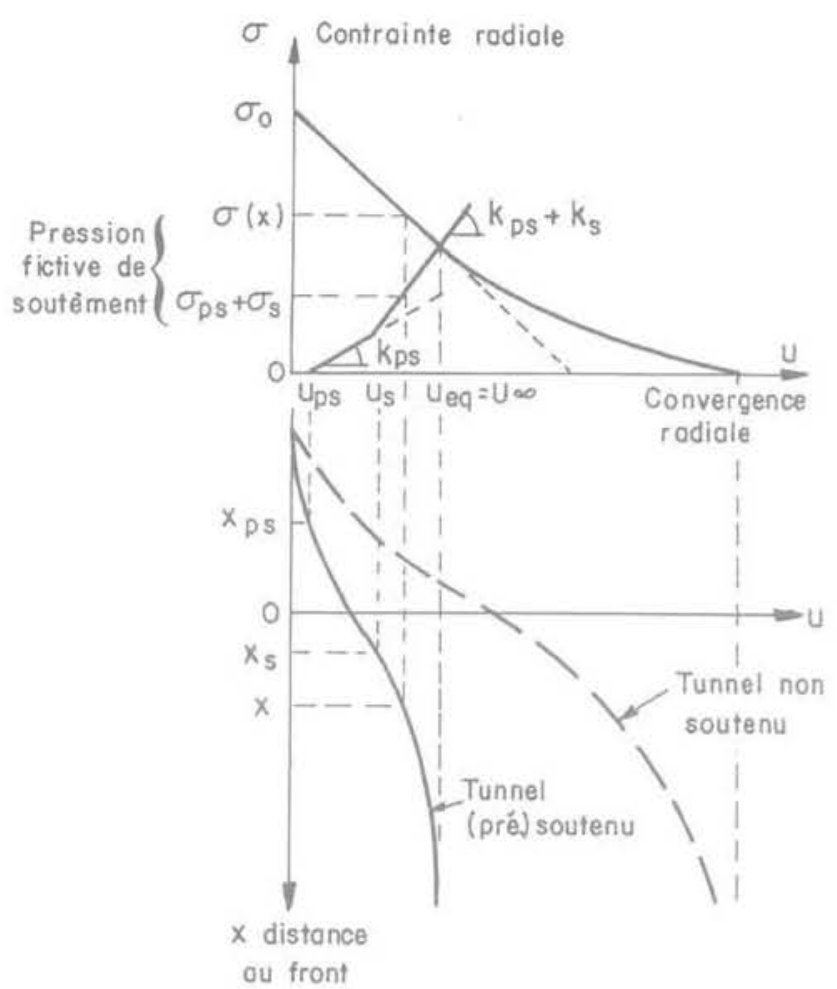

FG. 2 Étude du creusement d'un tunnel à l'aide d'une modélisation bidimensionnelle. Analysis of tunnel excavation by 2D-modelisation.

- le cas d'un tunnel soutenu « traditionnellement » en arrière du front a ensuite été étudié, conduisant à des méthodes telles que la Nouvelle Méthode Implicite (Bernaud et al., 1992 et 1994) ;

- l'objet de cet article est de présenter des résultats obtenus récemment dans le cas de mise en place de pré-soutènements (en avant du front).

\section{Quelques remarques sur les méthodes de pré-soutènement}

Il existe dans la pratique diverses méthodes de présoutènement, qui sont le plus souvent associées à des soutènements classiques (cintres, béton projeté, boulons) mis en place en arrière du front. Ces pré-soutènements peuvent dans certains cas être combinés entre eux. On ne décrira pas ici en détail les différentes méthodes utilisées, mais on se limitera à en donner les principales caractéristiques ayant une incidence sur le comportement.

\section{- La mise en place d'une voûte-parapluie}

C'est une méthode consistant à introduire dans le sol à partir du front de taille des barres ou tubes métalliques quasi parallèles à l'axe du tunnel (inclinaison de 8 à $10^{\circ}$ par rapport à $1^{\prime}$ horizontale), sur des longueurs de 10 à 15 m en général, avec un recouvrement entre deux voûtes successives de l'ordre de 2 à $5 \mathrm{~m}$. Les tubes peuvent être soit foncés, soit scellés au terrain par injection de coulis. Au fur et à mesure de l'avancement, le dis- 
positif est complété par la mise en place d'un soutènement souvent constitué de cintres et béton projeté.

La voûte-parapluie permet en pratique de protéger la zone de décousu en reportant les charges d'une part sur le terrain en avant de l'excavation et d'autre part sur le soutènement en arrière (report de charges longitudinal). Étant constituée d'éléments qui ne sont pas complètement solidarisés transversalement, elle ne peut pas en général être considérée comme constituant une coque (peu de report de charges transversal), et elle est par conséquent associée à un soutènement «traditionnel $»$.

\section{- La méthode de pré-découpage mécanique et} pré-voûte

Cette méthode consiste à réaliser à l'avancement, à l'aide d'une haveuse, une saignée périphérique épousant la ligne d'extrados du futur ouvrage. Cette saignée est immédiatement remplie de béton projeté à haute résistance aux jeunes âges. Après durcissement du béton, la coque protectrice (pré-voûte) assure le soutènement de la travée en cours de creusement, ce qui permet de l'associer de plus en plus souvent à un terrassement en pleine section, avec en général un renforcement du front.

L'épaisseur de la saignée est de l'ordre de 15 à $25 \mathrm{~cm}$, sa longueur est d'environ 4 à $5 \mathrm{~m}$. Le recouvrement entre deux éléments tronconiques successifs est variable suivant la nature du terrain, mais le plus souvent de l'ordre de 0,5 à $1 \mathrm{~m}$.

\section{- Réalisation de colonnes de jet-grouting hori- zontales}

Cette méthode consiste à réaliser à l'avancement des zones tronconiques de terrain traité grâce à la réalisation de colonnes de jet-grouting quasi parallèles à l'axe du tunnel, de diamètre pouvant varier entre 0,5 et $0,8 \mathrm{~m}$. En réalisant des colonnes sécantes, on peut créer une coque continue, dont l'épaisseur peut être augmentée en multipliant le nombre d'auréoles, et ainsi obtenir un effet de voûte artificiel même dans des terrains totalement dépourvus de cohésion. Le principe de fonctionnement est le même que pour une voûte-parapluie, mais avec un report de charges tranversal bien meilleur, dans la mesure où l'on peut considérer que les auréoles constituent une coque continue.

- Renforcement du front de taille par des boulons en fibre de verre

Cette méthode est particulièrement adaptée aux terrains susceptibles d'extrusion ou dans le cas d'excavation en pleine section amenant une surface du front de taille importante. Elle peut dans ce cadre être associée à d'autres méthodes de pré-soutènement. Elle consiste à mettre en place parallèlement à l'axe du tunnel une série de boulons en fibre de verre scellés au coulis de ciment dans des forages préalables. Ces boulons allient une résistance très élevée à une grande fragilité ce qui permet de les briser facilement avec l'outil d'excavation. La longueur des boulons, principalement limitée par les conditions de mise en œuvre, est en général de 15 à $20 \mathrm{~m}$, avec un recouvrement entre deux séries successives de boulons.

Toutes ces techniques ont pour points communs de venir renforcer le terrain au niveau du front de taille, voire en avant de celui-ci. Elles participent de manière très efficace à la stabilisation de la zone particulièrement sensible du front et par conséquent à la stabilisation de l'ensemble de l'ouvrage. Il faut enfin noter que leur mise en place permet en général de limiter de façon très sensible les convergences de l'excavation au niveau du front comme très loin de celui-ci. Elles sont donc particulièrement bien adaptées au cas des excavations réalisées dans des terrains meubles et instables.

Elles ne sont cependant pas équivalentes et l'on peut effectuer des distinctions selon plusieurs critères : - l'étendue de la zone affectée peut être sensiblement différente selon les techniques : une voute-parapluie ou des colonnes de jet-grouting pourront intervenir loin en avant du front (une distance pré-soutenue de l'ordre de $15 \mathrm{~m}$ est tout à fait courante). Une pré-voûte ne pourra pas quant à elle s'avancer à plus de 4 ou $5 \mathrm{~m}$ en avant du front : son action sera par conséquent limitée à la zone du front :

- la distance pré-soutenue sera par conséquent considérée comme variable lorsque plusieurs phases d'excavation peuvent être réalisées entre deux mises en place successives du pré-soutènement (voûte-parapluies ou jet-grouting). Elle pourra en première approche être considérée comme constante dans les autres cas (prévoûte)

- les modes de fonctionnement mécanique des divers types de pré-soutènements sont divers : lorsque les différents éléments constituant le pré-soutènement sont solidaires entre eux (éléments de pré-voûte, colonnes de jet-grouting avec un certain recouvrement...), leur comportement peut être modélisé par celui d'une coque. Dans le cas d'une voûte-parapluie, le comportement de la structure sera beaucoup mieux approché en considérant des éléments de poutre reposant sur le terrain non excavé d'une part et sur le soutènement mis en place en arrière du front d'autre part. On pourra. néanmoins, effectuer une analyse intéressante en axisymétrie en considérant une zone de terrain améliorée par la présence de la voûte-parapluie, notamment lorsque les tubes sont scellés au terrain par injection sous forte pression. On effectuera ici l'étude en considérant pour cette zone des caractéristiques moyennes tenant compte de la présence des éléments de renforcement selon un principe d'homogénéisation (Fig. 3).

Un renforcement du front par des boulons en fibre de verre met, quant à lui, en jeu des mécanismes fondamentalement différents où il convient de considérer

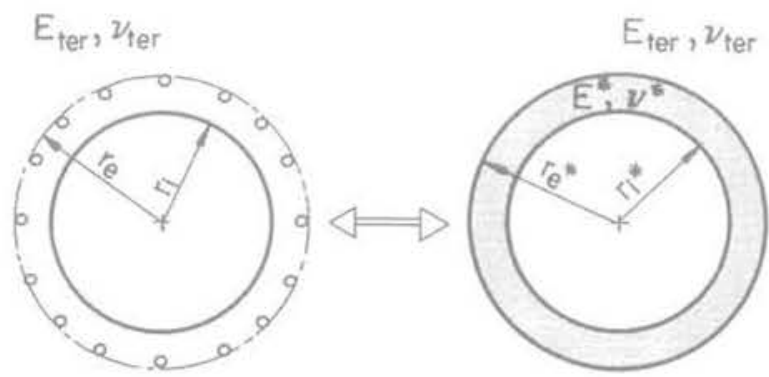

$$
\begin{aligned}
& \sum E_{1} \cdot S_{1}=E^{*} \cdot S^{*} \\
& \sum E_{1} \cdot I_{1}=E^{*} \cdot I^{*}
\end{aligned}
$$


un volume de terrain traité. L'action de ce dernier type de pré-soutènement ne sera pas envisagée dans le présent article,

On distinguera donc fondamentalement ici deux types de pré-soutènements :

- les pré-soutènements de type 1, qui intéressent un volume de terrain important et viennent agir loin en avant du front. Il s'agit en particulier de la voûte-parapluie et des colonnes de jet-grouting horizontales. Il faut alors tenir compte ici de l'épaisseur du terrain traité et la longueur pré-soutenue ne peut être considérée comme constante. On a en outre considéré dans ce cas que la mise en place d'un tel pré-soutènement formait une couronne de terrain traité (coque) de caractéristiques homogènes équivalentes ;

- les pré-soutènements de type 2, dont l'action est localisée au voisinage du front, c'est-à-dire ceux qui intéressent un volume de terrain relativement faible. Il s'agit typiquement de la pré-voûte. Compte tenu de la faible épaisseur du pré-soutènement, on a assimilé la surface de contact entre le pré-soutènement et le terrain à la surface d'excavation. De plus, dans la mesure où un élément de pré-voûte est en général réalisé à chaque phase d'excavation, on a considéré la lonqueur pré-soutenue comme constante.

\section{4}

\section{Approches usuellement utilisées dans le cas de la mise en place de soutènements classiques}

Il est intéressant à ce niveau de préciser les hypothèses et le domaine de validité des diverses méthodes dérivant de la méthode convergence-confinement afin de mettre en évidence la nature des études complémentaires à effectuer pour estimer l'action des pré-soutènements.

Le principe de ces méthodes ne sera pas décrit en détail ici mais a fait l'objet d'un certain nombre de publications citées en bibliographie. Ces méthodes fournissent une estimation de la convergence $u(x)$ à une certaine distance $\mathrm{x}$ en arrière du front à partir de la donnée (explicite ou non) de la convergence de l'excavation à l'endroit de la pose du soutènement. La détermination de celle-ci repose sur l'évaluation de

- la convergence au front $\mathrm{u}(0)$,

- la convergence loin en arrière du front $\mathrm{u}_{z}$;

- une fonction dite fonction de forme a(x) définie pour $x>0$ (en arrière du front) par la relation:

$$
a(x)=\frac{u(x)-u(0)}{u_{-}-u(0)}
$$

On peut alors citer trois méthodes applicables en théorie:

- La méthode convergence-confinement « classique 》 : elle utilise les valeurs de $u(0)$ et a(x) déterminées dans le

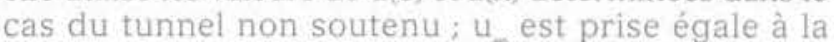
convergence à l'infini du tunnel non soutenu;

- la «Méthode implicite b: elle utilise encore les valeurs de $u(0)$ et $a(x)$ déterminées dans le cas du tunnel non soutenu; mais $\mathrm{u}_{\text {s }}$ est prise égale à la convergence à l'infini du tunnel soutenu :

- la a Nouvelle Méthode implicite n: la valeur de u(0) reste calculée à partir du cas du tunnel non soutenu ; mais la fonction de forme $\mathrm{a}^{\mathrm{s}}(\mathrm{x})$ se calcule à partir de la fonction de forme du tunnel non soutenu $a(x)$ en utilisant la relation $\mathrm{a}^{\mathrm{s}}(\mathrm{x})=\mathrm{a}(\alpha . \mathrm{x})$ où le coefficient $\alpha$ (fonction de soutènement) ne dépend que de la raideur du soutènement. $u_{\text {u }}$ est prise égale à la convergence à l'infini du tunnel soutenu.

Il convient néanmoins de noter que ces approches : - ne permettent pas de décrire les phénomènes se produisant en avant du front,

- ne tiennent pas compte de la convergence réelle au niveau du front, et notamment de l'influence de la distance de pose du soutènement,

et ne sont en tout cas pas dírectement applicables à l'étude de la mise en place de pré-soutènements ni même dans un certain nombre de cas où un soutènement classique est mis en place très près du front.

\section{5}

\section{Méthode d'étude}

Afin d'étudier l'influence des pré-soutènements sur la courbe de convergence en fonction de la distance au front, on a effectué une série de calculs numériques par éléments finis sur un modèle axisymétrique. Le code utilisé était CESAR, développé par le LCPC, avec le maillage représenté sur la figure 4.

Les hypothèses associées à ce type de calcul sont celles relatives à l'utilisation de la méthode convergence-confinement, correspondant en fait aux tunnels creusés à grande profondeur :

- une géométrie de l'excavation pas trop éloignée de l'axisymétrie :

- un massif de terrain homogène et isotrope ;

- les types de comportement du terrain envisagés ici sont un comportement élastique linéaire ou élasto-plastique parfait avec le critère de Tresca, dont quelques cas ont été envisagés.

Il apparaît en pratique qu'une étude peut être effectuée en faisant varier l'ensemble des paramètres adimensionnels indépendants suivants :

$\mathrm{U}=\mathrm{u} / \mathrm{r}$ convergence $\mathrm{u}$ rapportée au rayon $\mathrm{r}$ de l'excavation.

En élasticité :

$\sigma_{0}^{\prime}=\sigma_{0} / \mathrm{E}$ contrainte initiale régnant dans le terrain $\sigma_{i}$ rapportée au module d'Young $\mathrm{E}$ de celui-ci :

$v \quad$ coefficient de Poisson du terrain ;

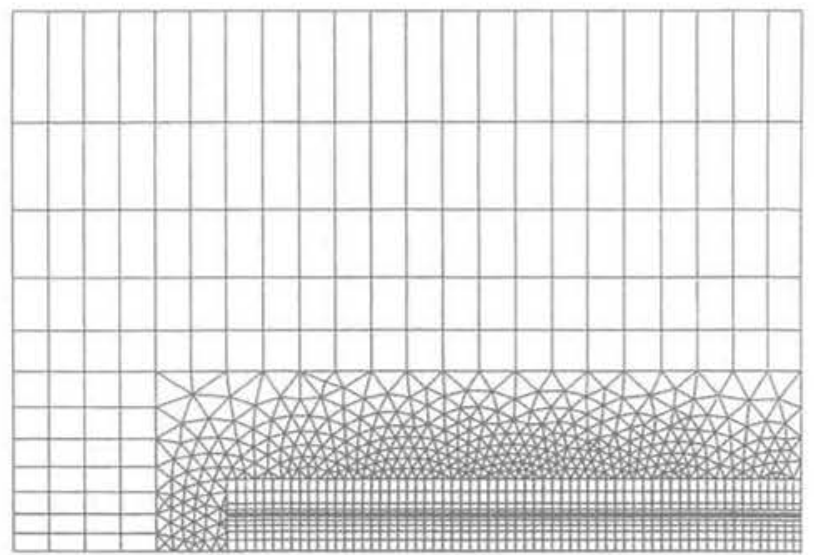

FIG.4 Maillage utilisé.

FEM mesh used for the calculations. 
$\mathrm{K}_{\mathrm{s}}^{\prime}=\mathrm{K}_{\mathrm{s}} / \mathrm{E}$ rigidité du soutènement $\mathrm{K}_{\mathrm{s}}$ rapportée au module d'Young du terrain $\mathrm{E}$;

$\mathrm{d}_{0}^{\prime}=\mathrm{d}_{0} / \mathrm{r}$ distance de pose du soutènement au front de taille $\mathrm{d}_{0}$ rapportée au rayon $\mathrm{r}$ de l'excavation. On considérera qu'une valeur positive de $d_{0}$ ' correspond à la distance de pose au front d'un soutènement classique et qu'une valeur de $\mathrm{d}_{0}$ ' négative correspond à une distance pré-soutenue, ce qui permet une relation de continuité entre des méthodes a priori fondamentalement différentes comme le soutènement d'un tumnel par projection de béton en arrière du front et la mise en place d'une pré-voûte.

\section{En plasticité}

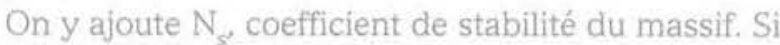
le comportement du massif suit une loi de type Tresca, $\mathrm{N}_{\mathrm{s}}=\sigma_{0} / \mathrm{c}_{\mathrm{u}}$ est égal au rapport de la contrainte initiale $\sigma_{0}$ régnant dans le massif sur la cohésion $c_{u}$ de celui-cí.

La méthode d'étude s'articule autour de trois étapes:

- l'estimation de la convergence au niveau du front; - l'estimation de la forme de la courbe de convergence en avant du front, et donc la détermination de la convergence en tout point en avant du front ;

- la détermination de la convergence en arrière du front par adaptation des méthodes classiques.

Le lien entre la convergence de l'excavation et la contrainte (réelle ou fictive) appliquée sur la surface de celle-ci est effectué à l'aide de la loi de comportement du terrain (symbolisée par la courbe de terrain dans la méthode convergence-confinement).

Le principe de la démarche utilisée consiste à réaliser des études paramétriques en utilisant les calculs éléments finis en axisymétrie, puis à approcher les résultats obtenus par des formulations analytiques reproduisant au mieux les résultats des calculs numériques. On présente en annexe l'essentiel des résultats détaillés obtenus (résultats des calculs numériques et calage par formules analytiques). Une synthèse des principaux résultats, avec les formulations analytiques, et de leur utilisation pratique est décrite ci-après.

\section{Résultats obtenus en élasticité}

En premier lieu, il est intéressant de présenter la figure 5 , qui montre les courbes convergence/distance au front obtenues pour différentes distances de pose $\mathrm{d}_{0}^{\prime}$ du soutènement, à partir des calculs numériques dans un cas particulier ( $\left.\mathrm{v}=0,48 ; \sigma_{i j}^{\prime}=0,006 ; \mathrm{K}_{\mathrm{s}}^{\prime}=7\right)$. On observe très bien que les convergences augmentent avec d' notamment entre les valeurs négatives (présoutènements) et les valeurs positives (soutènements).

\section{1}

\section{Convergence au niveau du front $\mathrm{U}(0)$}

\section{1 .1}

\section{Cas du tunnel non soutenu}

Ce cas a largement été étudié dans la pratique. Dans le cadre de cette étude, il nous a paru intéressant de préciser l'influence du coefficient de Poisson sur la convergence au front. Les résultats obtenus sont présentés sur la figure 6, qui montre que cette influence,

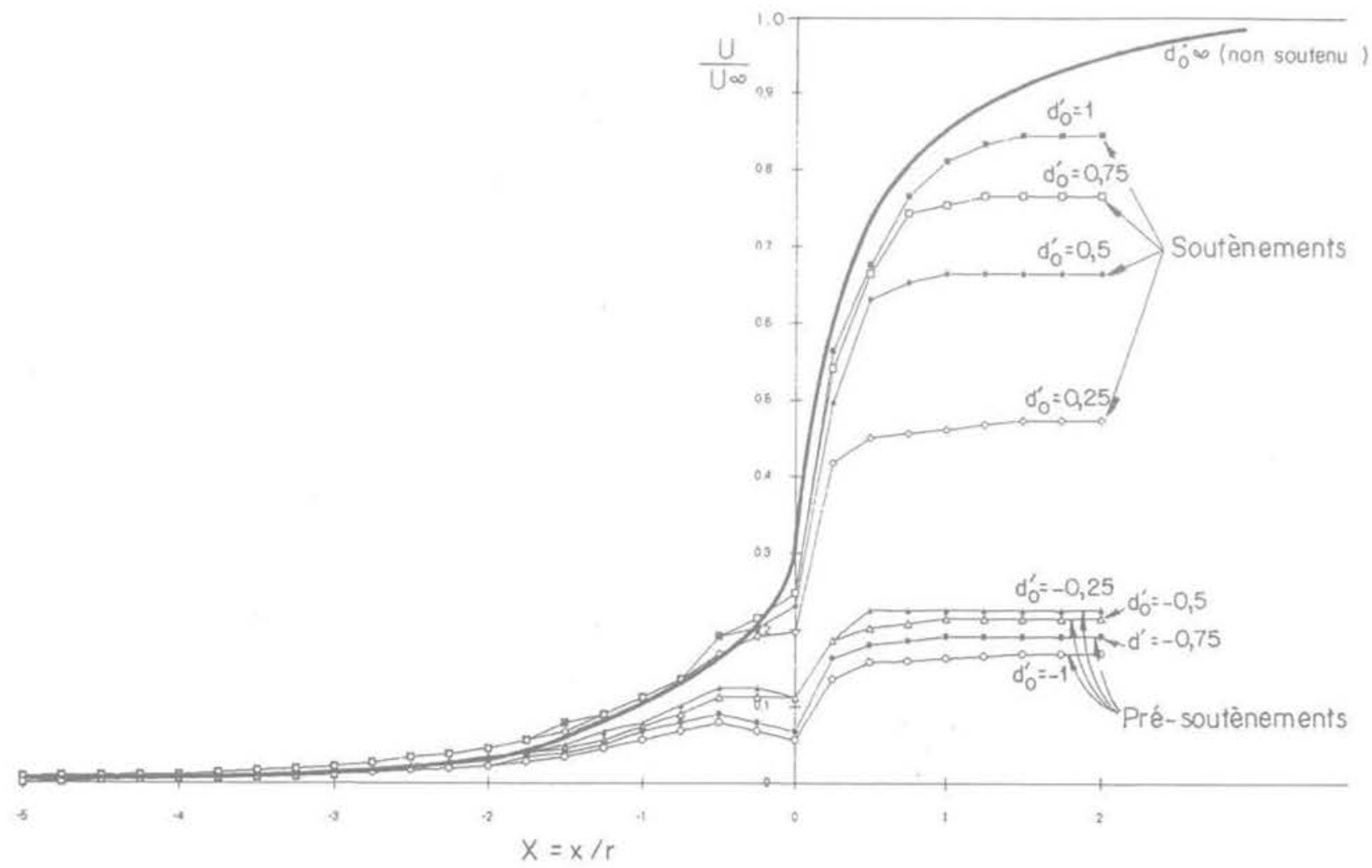

FG.5 Courbes de convergence en fonction de la distance de pose $\mathrm{d}^{\prime}$. Convergences curves with respect to lining placement distance $\mathrm{d}_{\mathrm{o}^{\prime}} \mathrm{d}^{\prime}$ 


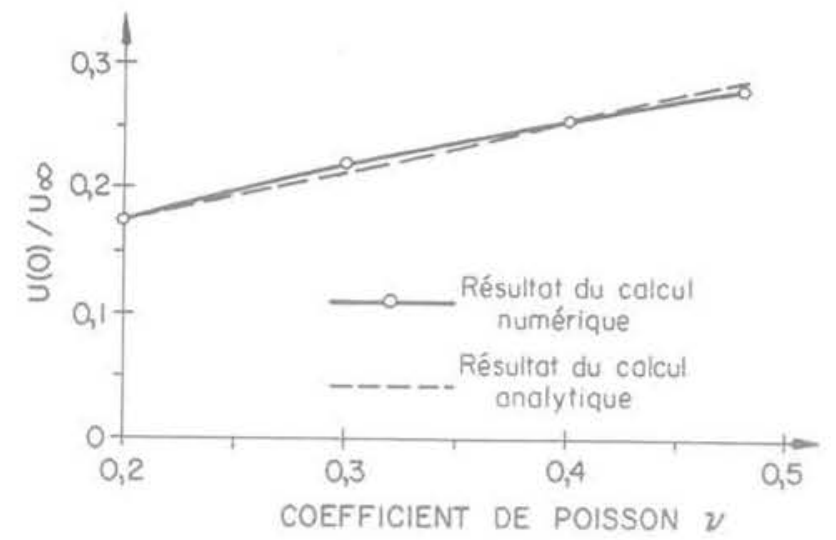

FG. 6 Influence du coefficient de Poisson sur la convergence au front.

Influence of the Poisson ratio on the convergence at the face.

souvent négligée en pratique, peut parfois être significative. Elle peut être approchée par la formule suivante:

$$
\frac{\mathrm{U}(0, v)}{\mathrm{U}_{-}}=0,4 \mathrm{v}+0,095
$$

où $\mathrm{U}_{\infty}$ désigne la convergence à l'infini du tunnel non soutenu :

$$
\mathrm{U}_{-}=(1+v) \cdot \sigma_{0}{ }^{\circ}
$$

Dans toute la suite de l'étude, on se limitera au cas $v$ $=0,48$ (proche de 0,5 ), qui conduit à la relation usuelle $\mathrm{U}(0)=0,287 \mathrm{U}_{\infty}$

\section{$6.8,2$}

Cas de la mise en place d'un pré-soutènement de type 2 (pré-voûte ou soutènement classique par mise en place de béton projeté)

L'étude réalisée dans ce cas conduit à déterminer la convergence au front dans les deux cas extrêmes suivants :

- le cas ou le tunnel n'est pas soutenu : on se ramène alors au cas précédent. La valeur de la convergence au front dans ce cas sera notée $\mathrm{U}(0,+\infty)$;

- le cas ou le pré-soutènement s'avance à une distance infinie en avant du front. La convergence au front dans ce cas sera notée $U(0,-\infty)$, qui peut s'exprimer par la relation suivante (cf. annexe A11):

$$
U(0,-\infty)=\frac{(1+v) \cdot \sigma_{0}}{(1+v) \cdot K_{s}+1} \cdot\left(0,74-\frac{1}{\left(K_{s}+3\right)^{0,7}}\right) \cdot \frac{0,4 \cdot v+0,095}{0,287}
$$

Dans les cas intermédiaires, où la distance de pose du (pré-)soutènement a une valeur finie $d_{0}^{\prime}$, la convergence au front de taille $\mathrm{U}\left(0, \mathrm{~d}_{0}\right)$ se situe intuitivement entre les deux valeurs calculées précédemment. Une loi de variation de la forme:

$$
\mathrm{U}(0)=f\left[\mathrm{U}(0,-\infty), \mathrm{U}(0,+\infty), \mathrm{d}_{0}{ }^{\prime}\right]
$$

peut s'exprimer sous la forme suivante (cf. annexe A12):

$$
U\left(0, d_{0}\right)=\frac{U(0,+\infty)-U(0,-\infty)}{\pi} \cdot \operatorname{Arctan}\left(2,8 \cdot d_{0}-0,3\right)+\frac{U(0,+\infty)+U(0,-\infty)}{2}
$$

\section{4., 3}

Cas de la mise en place d'un pré-soutènement de type 1 (voûte-parapluie ou de colonnes de jet-grouting)

Comme il a été noté plus haut, ces formes de présoutènement interviennent loin en avant du front ce qui ne permet plus de considérer la distance pré-soutenue comme constante. Les résultats numériques ont montré que la convergence au front était fortement atténuée dans le cas de la mise en place de telles formes de présoutènements. On pourra donc utiliser en première approximation la valeur de la convergence du tunnel infiniment soutenu, déterminé selon la formule du paragraphe 6.1.2:

$$
\mathrm{U}(0)=\mathrm{U}(0,-\infty)
$$

\section{2}

\section{Convergence en avant du front}

De façon très générale, la convergence en avant du front peut être très bien approchée par une fonction de forme $b(x)$ du type :

$$
\mathrm{b}(\mathrm{x})=\frac{\mathrm{U}(\mathrm{x})}{\mathrm{U}(0)}=\frac{1}{1-\beta \cdot \mathrm{x}+\mathrm{x}^{2}}
$$

oủ $\beta\left(v, K_{s^{\prime}}^{\prime} d_{0}^{\prime}\right)$ dépend des paramètres du problème.

Des indications quant à la détermination de ce coef-

\begin{tabular}{|c|c|c|c|c|c|c|c|c|c|c|}
\hline \multirow{2}{*}{$\mathrm{K}^{\prime}$, } & \multicolumn{10}{|c|}{ Distance de pose $d^{\prime}$} \\
\hline & $-\infty$ & -1 & $-3 / 4$ & $-1 / 2$ & $-1 / 4$ & $1 / 4$ & $1 / 2$ & $3 / 4$ & 1 & $+\infty$ \\
\hline 0,35 & 0,6 & & & & & & & & & 0,6 \\
\hline 0,7 & 0,3 & 0,3 & 0 & 0,5 & 0,4 & 0,3 & 0,2 & 0,4 & 0,6 & 0,6 \\
\hline 7 & $-0,2$ & -1 & -1 & $(-0,8)$ & $-0,65$ & $-0,2$ & 0,1 & 0,25 & 0,2 & 0,6 \\
\hline 35 & & & & & & & & & & $-0,6$ \\
\hline
\end{tabular}
ficient $\beta$ sont données en annexe A2. On retiendra qu'il varie en fonction du coefficient de Poisson $v$ selon une loi du type suivant, pour le tunnel non soutenu:

$$
\beta(v)=\ln \left(-9 \cdot v^{2}+10 \cdot v-0,9\right)
$$

qui conduit, pour $v=0,48$, à la valeur usuelle de $\beta=0,6$.

TABLEAUI Valeurs de $\beta\left(\mathrm{K}_{\mathrm{s}^{\prime}} \mathrm{d}^{\prime}{ }_{\mathrm{o}}\right)$ pour $\mathrm{v}=0,48$. 
Cette fonction $\beta$ dépend également des caractéristiques du (pré-)soutènement (raideur K' et distance de pose $d_{2}^{\prime}$ ). Aucune loi générale n'a pu être définie, mais quelques résultats sont présentés dans le tableau I qui peuvent servir de guide pour le choix de valeurs de références.

La détermination de cette loi $\beta\left(v, K^{\prime}, d^{\prime}\right)$, associée à l'estimation de la convergence au front $\mathrm{U}(0)$, permettent de déterminer la valeur de la convergence en tout point situé en avant du front.

\section{3}

\section{Convergence en arrière du front}

Les étapes précédentes permettent de déterminer les valeurs de la convergence au niveau du front et en avant de celui-ci. On peut en particulier obtenir la valeur de la convergence à l'endroit de la pose d'un éventuel pré-soutènement. Pour déterminer la convergence loin en arrière du front, on propose la démarche suivante :

- dans le cas de la mise en place d'un soutènement classique, une évaluation précise de la convergence au front associée à l'application de la Nouvelle Méthode Implicite (NMI) fournit des résultats extrêmement proches de ceux obtenus par les calculs numériques.

$$
a(X)=\frac{U(X)-U(0)}{U_{-}-U(0)}=1-\left(\frac{0,84}{\alpha \cdot X+0,84}\right)^{2}
$$

où le coefficient $\alpha\left(\mathrm{K}^{\prime}\right)$ dépend de la rigidité du soutènement selon une loi du type (Bernaud, Rousset, 1992) :

$$
\alpha=1+0,635 \cdot K_{s}-0,0293 \cdot K_{s}^{2}+0,781 \cdot 10^{-3} \cdot K_{s}^{3}-0,64 \cdot 10^{-5} \cdot K_{,}^{\prime 4}
$$

- dans le cas des pré-soutènements, il paraît préférable de retenir une approche de type convergence-confinement (CVCF), fondée sur la détermination préalable de la valeur de la convergence au moment de la mise en place du pré-soutènement $\left.\mathrm{U}_{\left(\mathrm{d}_{0}^{\prime}\right)}\right)$ telle qu'exposée en 6.2 , et qui permet notamment de déterminer la convergence à l'équilibre loin en arrière du front $\mathrm{U}_{\infty}$ :

$$
\mathrm{U}_{-}=\frac{\sigma_{\mathrm{s}}+\mathrm{K}_{\mathrm{s}} \cdot \mathrm{U}\left(\mathrm{d}_{\mathrm{o}}\right)}{\mathrm{K}_{\mathrm{s}}+\frac{1}{1+v}}
$$

- la détermination de la convergence en tout point situé à l'arrière du front (en particulier la convergence lors de la pose du soutènement) peut être utilisée en utilisant par exemple la fonction de forme $a(x)$ du type :

$$
\mathrm{a}(\mathrm{X})=\frac{\mathrm{U}(\mathrm{X})-\mathrm{U}(0)}{\mathrm{U}_{-}-\mathrm{U}(0)}=1-\left(\frac{0,84}{\mathrm{X}+0,84}\right)^{2}
$$

Quelques comparaisons entre les résultats des calculs numériques et les résultats des formulations analytiques sont présentées en annexe A3.

\section{4}

\section{Mise en place de plusieurs formes de (pré-)soutènements de natures différentes}

Comme il a été signalé plus haut, la mise en place de pré-soutènements est généralement complétée par la mise en place d'autres types de soutènements. Lorsque l'épaisseur caractérisant chacun des types de (pré-)soutènement est faible devant le rayon d'excavation, on peut considérer que l'interface entre le massif de terrain et les différentes structures mises en place est unique. En revanche, pour des pré-soutènements traitant le terrain en profondeur (voûte-parapluie, colonnes de jet-grouting), cette hypothèse n'est plus valable. On propose en annexe A4 un principe de calcul pour la prise en compte de la mise en place simultanée de soutènements de type 1 et de type 2 . Ce principe est ensuite illustré sur un cas particulier.

\section{7}

\section{Limite du domaine élastique}

Dans le cas de la mise en place de pré-soutènements dans un massif de terrain au comportement élasto-plastique, il apparait que la validité d'une étude fondée sur l'hypothèse d'un comportement élastique du terrain ne se limite pas aux seuls cas où le coefficient de stabilité $N$, reste inférieur à 1. En effet, l'objectif est alors de maintenir le terrain le plus possible en état élastique : le fait d'intervenir pour confiner le terrain en avant du front peut lui permettre de conserver un comportement élastique mème lorsque ce coefficient de stabilité atteint des valeurs importantes.

On distingue en réalité deux types de zones plastifiées susceptibles d'apparaître autour d'un tunnel (Fig. 7) :

- une zone plastifiée située au voisinage du front qui apparaît systématiquement même pour des valeurs très faibles du coefficient de stabilité. Celle-ci se « déplace » avec le front et ses dimensions restent par conséquent limitées :

- une zone plastifiée située autour de l'excavation qui subsiste une fois le front très éloigné.

Il est particulièrement intéressant de déterminer les valeurs des paramètres pour lesquelles cette seconde zone n'apparait pas. On peut en particulier réaliser cette étude en utilisant les résultats obtenus en élasticité et en se plaçant à la limite d'un tel comportement: il suffit de déterminer la valeur $\mathrm{K}_{\text {s. plu }}(-\infty)$ pour laquelle un tunnel " infiniment pré-soutenu n ne verra pas l'apparition d'une zone plastifiée rémanente. En pratique on détermine $\mathrm{K}^{\prime} \quad(-\infty)$, pour un pré-soutènement infiniment long, de façon à ce que la valeur de la convergence à l'équilibre U soit égale à la valeur de la convergence $\mathrm{U}_{\mathrm{f}}$ correspondant à la limite entre le domaine élastique et le domaine plastique (cf. figure $8 \mathrm{a}$ ). On obtient ainsi la relation suivante:

$$
K_{\text {s.pis }}(-\infty)=\frac{N_{3}-1}{1+v}
$$

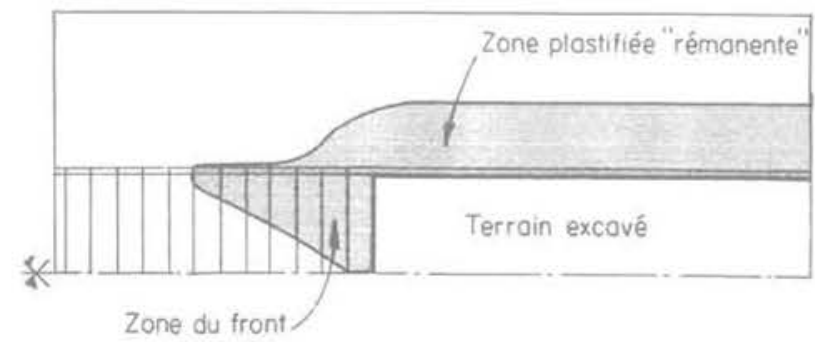

FG.7 Apparition des différentes zones plastifiées. Location of different plastic zones. 

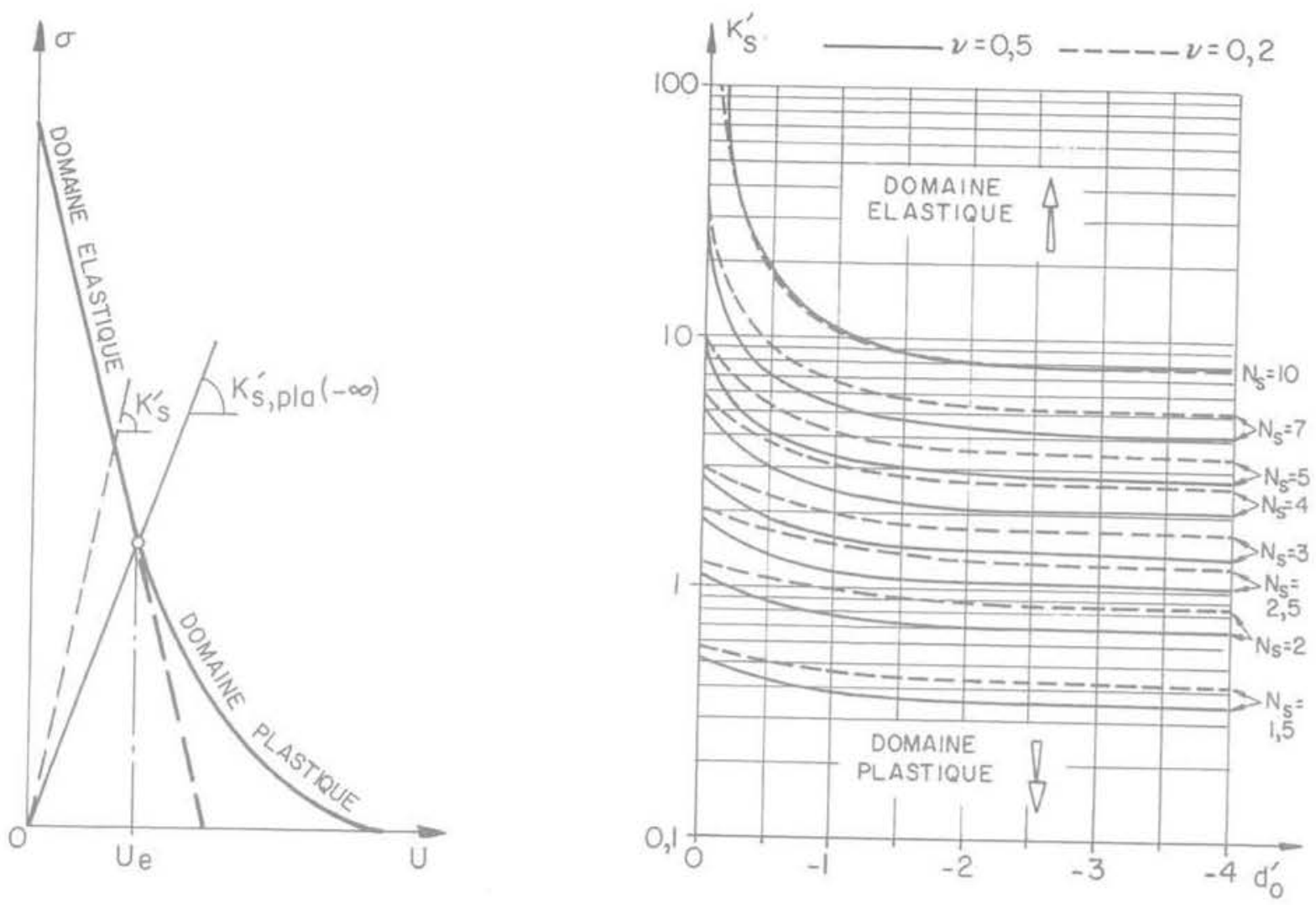

FG. 8 Limite du comportement élastique du terrain. Limits of the elastic behaviour of the ground.

et la relation entre $K_{\text {spla }}^{\prime}$ et la distance de pose du présoutènement $d_{0}^{\prime}$ est traduite par un abaque permettant de prévoir le type de comportement du terrain (cf. figure $8 \mathrm{~b}$, où la valeur de $\mathrm{K}_{\text {s.pla }}^{\prime}(-\infty)$ correspond à l'asymptote des différentes courbes) :

$-\mathrm{si}^{\prime}<\mathrm{K}_{\text {spla }}(-\infty)$ : il y aura une apparition d'une zone plastifiée quelle que soit la distance pré-soutenue : - si $\mathrm{K}^{\prime}$ s. $>\mathrm{K}_{\text {s.pia }}^{\prime}(-\infty)$ : l'apparition ou non d'une zone plastifiée dépendra de la distance pré-soutenue.

\section{8}

\section{Résultats obtenus en plasticité}

Enfin, un certain nombre de calculs effectués en considérant un comportement élasto-plastique du terrain dans le cas où une zone plastifiée apparaît autour de l'excavation a permis de mettre en évidence quelques tendances, illustrées par les figures présentées en annexe B :

- une moins grande dépendance des phénomènes se produisant en avant du front de taille en fonction de la variation du coefficient de Poisson et de la distance de pose ;

- l'apparition d'une grande part des déplacements en avant du front lorsque le coefficient de stabilité est élevé.

La démarche pratique proposée pour l'analyse du comportement des tunnels mettant en œuvre un présoutènement lorsqu'il y a apparition de zones plastifiées est la suivante:

- détermination de la convergence au front $\mathrm{U}(0)$ : elle dépend a priori, bien sùr, des mèmes paramètres qu'en

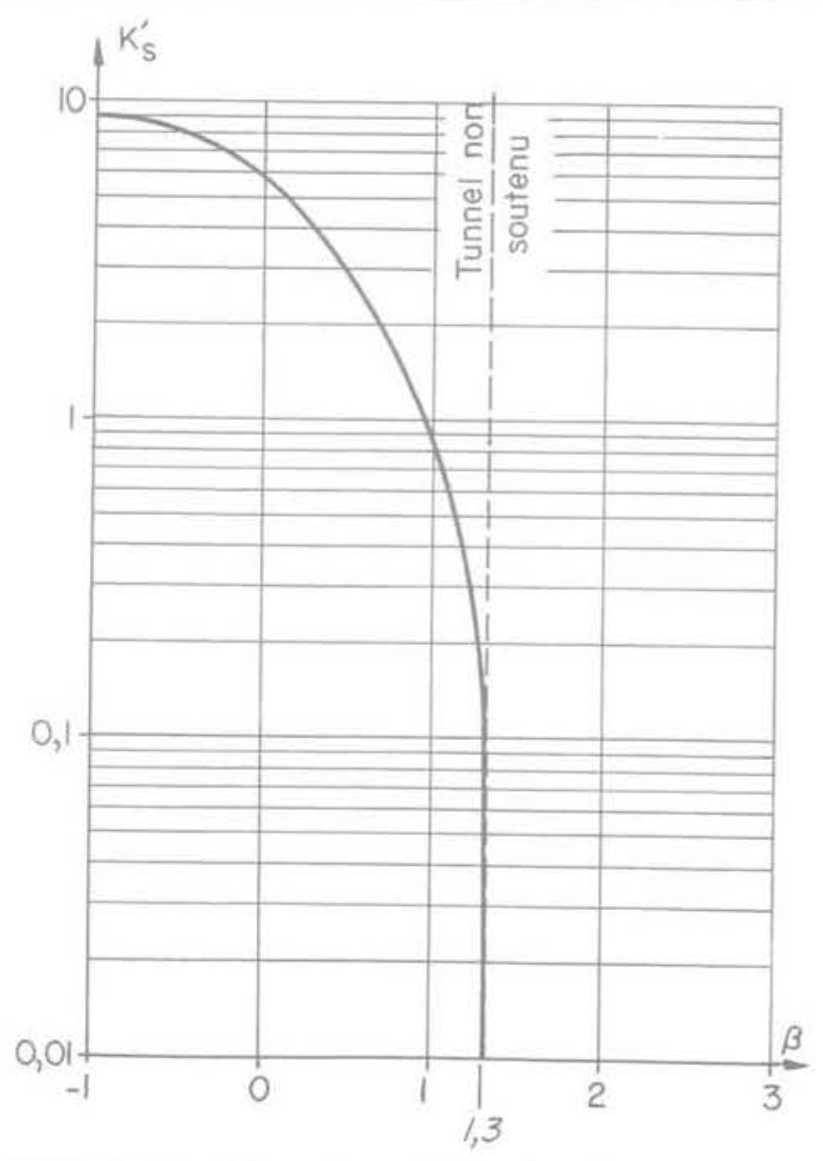

F6.9 Valeurs du coefficient $\beta$ en fonction de la raideur du soutènement $\mathrm{K}^{\prime}$.

Values of $\beta$ coefficient with respect to lining stiffness $\mathrm{K}^{\prime}$. 
élasticité, et en plus du facteur de stabilité $\mathrm{N}$. En pratique, on constate qu'elle dépend peu de $\mathrm{K}^{\prime}$, et reste en fait très proche, dans le domaine élasto-plastique, de la valeur élastique déterminée pour $\mathrm{K}_{\text {s, pia }}^{\prime}$;

- détermination de la convergence en avant du front: on utilise le même type de relation entre $\mathrm{U}(\mathrm{x})$ et $\mathrm{U}(0)$ qu'en élasticité (cf. paragraphe 6.2), avec une fonction de forme $b(x)$ dépendant très peu du coefficient de Poisson $v$ et de la distance de pose $\mathrm{d}^{\prime}{ }^{\prime}$ mais assez nettement de la raideur du soutènement $\mathrm{K}^{\prime}$, notamment lorsqu'elle est élevée : pour $\mathrm{K}_{\mathrm{s}}^{\prime}<0,1$, on peut retenir $\beta=$ 1.3; au-delà on retiendra une loi de variation de $\beta$ telle que représentée sur la figure 9 ;

- détermination de la convergence en arrière du front: on utilisera les mêmes méthodes qu'en élasticité (cf. $\S 6.3)$.

\section{9}

\section{Conclusion}

La présente étude a permis de confirmer la nécessité de déterminer la variation du taux de déconfinement ou de la convergence de la paroi d'un tunnel en fonction de la distance au front de taille, lorsqu'on souhaite effectuer un calcul numérique à l'aide d'un modèle bidimensionnel, et de décrire la démarche à adopter.

On a en outre mis en évidence les hypothèses d'application et les limites des méthodes liées à la méthode convergence-confinement, montrant clairement que ces méthodes ne sont pas directement applicables au cas de la mise en place de pré-soutènement. Ceux-ci ont par ailleurs pu être classés en plusieurs catégories en fonction de leurs caractéristiques géométriques et de leur mode de fonctionnement.

Un des avantages de l'utilisation des pré-soutènements est qu'ils permettent dans un grand nombre de cas de confiner suffisamment le terrain pour le maintenir dans une phase de comportement élastique, ceci même lorsque le facteur de plasticité est élevé. On a ainsi produit des abaques permettant de savoir si le terrain risquait ou non de se plastifier, compte tenu de ses caractéristiques et du type de pré-soutènement mis en place. Ces abaques peuvent être généralisés à l'ensemble des problèmes susceptibles d'être rencontrés en utilisant ces résultats obtenus en élasticité.

Que l'on se place en élasticité ou en élasto-plasticité la méthode d'étude proposée permet de généraliser les

\begin{tabular}{|c|c|c|c|}
\hline & $\begin{array}{c}\text { Soutènement } \\
\text { classique }\end{array}$ & $\begin{array}{c}\text { Pré soutenement } \\
\text { de type } 1 \\
\text { (voûte-parapluie) }\end{array}$ & $\begin{array}{l}\text { Pré-soutènement } \\
\text { de type } 2 \\
\text { (pré-yoûte) }\end{array}$ \\
\hline $\begin{array}{l}\text { Au front } \\
\text { de taille }\end{array}$ & $\begin{array}{l}-\infty) \\
x) \rightarrow U(0)\end{array}$ & $U(0)=U(0,-\infty)$ & $\begin{array}{l}\mathrm{U}(0,+\infty) \\
\mathrm{U}(0,-\infty) \rightarrow \mathrm{U}(0) \\
\mathrm{d}_{0}\end{array}$ \\
\hline $\begin{array}{l}\text { En avant } \\
\text { du front }\end{array}$ & & \multicolumn{2}{|l|}{ Evaluation de $\beta$} \\
\hline $\begin{array}{l}\text { En arrière } \\
\text { du front }\end{array}$ & $\begin{array}{l}\text { Convergence } \\
\text { au front } \\
\text { + NMI }\end{array}$ & \multicolumn{2}{|c|}{$\begin{array}{l}\text { Convergence à la pose } \\
\text { du pré-soutènement } \\
\text { + CVCF }\end{array}$} \\
\hline
\end{tabular}

NMI : nouvelle méthode implicite.

CVCF: méthode convergence-confinement.

résultats déjà acquis aux cas des pré-soutènements, notamment en ce qui concerne la convergence au front (en fonction du type de pré-soutènement mis en cuvre), et la forme de la courbe de convergence en avant du front. En pratique (cf. tableau II) :

- on commence par évaluer la valeur de la convergence au niveau du front de taille $\mathrm{U}(0)$ à partir de la distance de pose et de la raideur des (pré-)soutènements ; l'effet de la distance de pose notamment constitue, même pour les soutènements classiques, un apport vis-à-vis des méthodes existantes:

- puis on en déduit la courbe de convergence en avant du front à partir d'une fonction de forme $b(X)$, qui dépend essentiellement des distances de pose et raideurs des différents (pré-)soutènements ;

- enfin, on détermine la courbe de convergence en arrière du front de taille à partir des approches classiques (nouvelle méthode implicite ou méthode convergence-confinement selon les cas).

De plus nous avons présenté une méthode de détermination de la courbe de convergence du terrain lorsque plusieurs types de (pré-)soutènements sont mis en place, par combinaison des études précédentes.

Le travail à réaliser reste cependant important, tant pour mieux appréhender les phénomènes liés aux comportements élasto-plastiques et visco-plastiques (en particulier lorsque le coefficient de stabilité est élevé), pour modéliser de façon plus réaliste certaines formes de pré-soutènement, que pour tenir compte de géométries qui sont parfois très éloignées de l'axisymétrie.

\section{Bibliographie}

AFTES (Groupe de travail $n^{\circ} 7$ ) - Soutènement et revètement : emploi de la méthode convergence-confinement, Revue Tunnels et Ouvrages souterrains, $\mathrm{n}^{\circ}$ spécial, novembre 1984

Bernaud D., Corbetta F., Minh D.N. Contribution à la méthode convergence-confinement par le principe de similitude, Revue Française de Géotechnique, $n^{\circ} 54$, janvier 1991.

Bernaud D., Rousset G. - La a Nouvelle Méthode implicite no pour l'étude du dimensionnement des tunnels, Revue
Française de Géotechnique, $n^{\circ} 60$, juillet 1992

Bernaud D., Benamar I., Rousset G. - La (Nouvelle Méthode implicite » pour le calcul des tunnels dans les milieux élastoplastiques et visco-plastiques, Revue Francaise de Géotechnique, n 68, 1994.

Panet M. - Stabilité et soutènement des tunnels, in : La Mécanique des roches appliquée aux Ouvrages de Génie Civil, chapitre IX, Association Amicale des Ingênieurs Ancien Élèves de l'ENPC. 1976.
Panet M., Guenot A. - Analysis of convergence behind the face of a tunnel, International Symposium i Tunneling 82 , Brighton, 1982

Panet M. - Le calcul des tunnels par la méthode convergence-confinement. Presses de l'ENPC, 1995.

Sato J., Ito J. - Numerical analysis of the umbrella method for tunnel excavation, Journées AFTES de Toulon : Infrastructures souterraines de transport, Ed. Reith, 1993. 
Résultats obtenus en élasticité

\section{A1}

\section{Étude de la convergence au niveau du front}

AIIT

Variation de la convergence au front d'un tunnel " infiniment pré-soutenu n en fonction de la rigidité du soutènement:

$$
\frac{\mathrm{U}(0,-\infty)}{\mathrm{U}_{\mathrm{s}}}=\frac{\xi\left(\mathrm{K}_{,}, \sigma_{0}, v\right)}{(1+v) \cdot \mathrm{K}_{\mathrm{s}}+1}
$$

avec dans le cas $\mathrm{v}=0,48$ :

$$
\xi\left(K_{s}\right)=0,74-\frac{1}{\left(K_{s}+3\right)^{n .7}}
$$

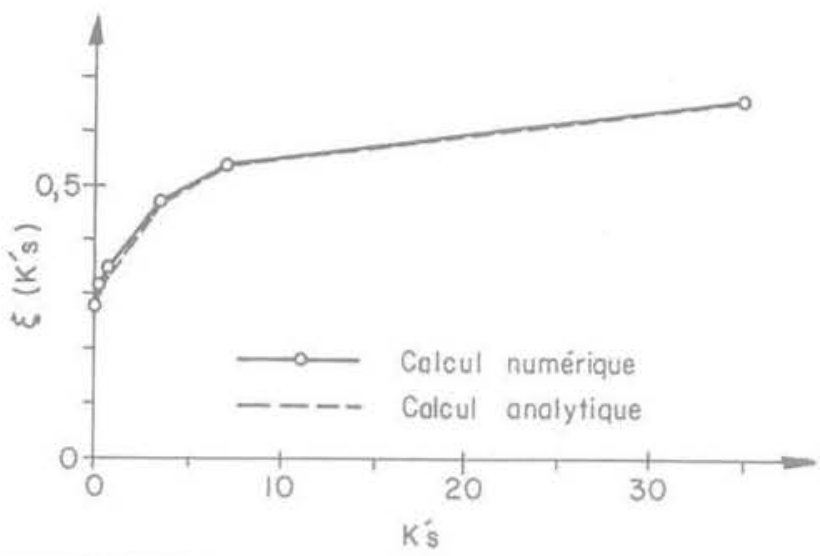

HG. AI1

On propose donc dans le cas général:

$$
\mathrm{U}(0,-\infty)=\frac{(1+v) \cdot \sigma_{0}}{(1+v) \cdot \mathrm{K}_{\mathrm{s}}+1} \cdot\left(0,74-\frac{1}{\left(\mathrm{~K}_{\mathrm{s}}+3\right)^{0,7}}\right) \frac{0,4 \cdot v+0,095}{0,287}
$$

Variation de la convergence au front d'un tunnel présoutenu ou simplement soutenu en fonction de la distance de pose du (pré-)soutènement:

$$
\begin{gathered}
\mathrm{U}\left(0, \mathrm{~d}_{0}\right)=\frac{\mathrm{U}(0,+\infty)-\mathrm{U}(0,-\infty)}{\pi} \cdot \operatorname{Arctan}\left(2,8 \cdot \mathrm{d}_{0}-0,3\right)+ \\
\frac{\mathrm{U}(0,+\infty)+\mathrm{U}(0,-\infty)}{2}
\end{gathered}
$$

- cas de la pré-voûte/soutènement par béton projeté $\left(v=0,48, \sigma_{0}^{\prime}=6.10^{-3}, K_{s}^{\prime}=7\right)$ (Fig. A12a);

- cas de la pré-voûte/soutènement par béton projeté $\left(v=0,48, \sigma_{0}^{\prime}=3.10^{-2}, K_{s}^{\prime}=0,7\right)$ (Fig. A12b).

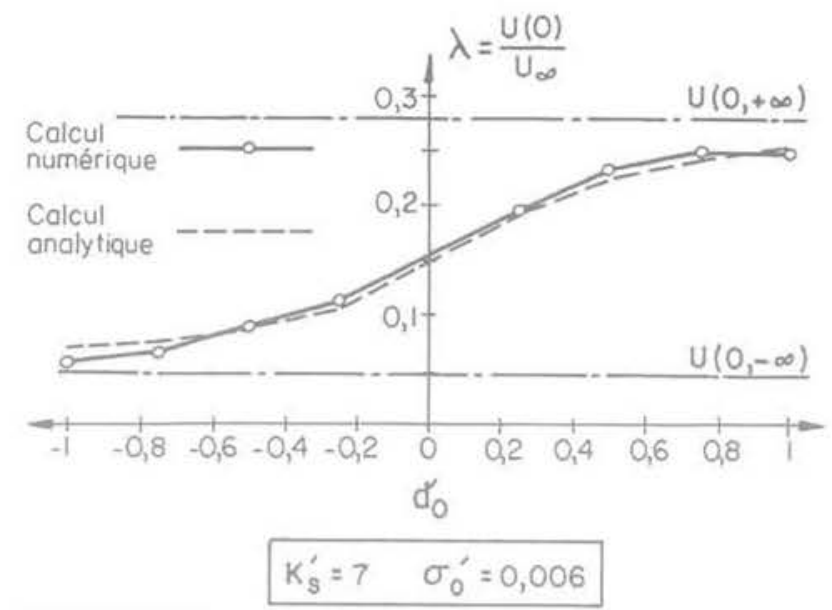

FG. A18

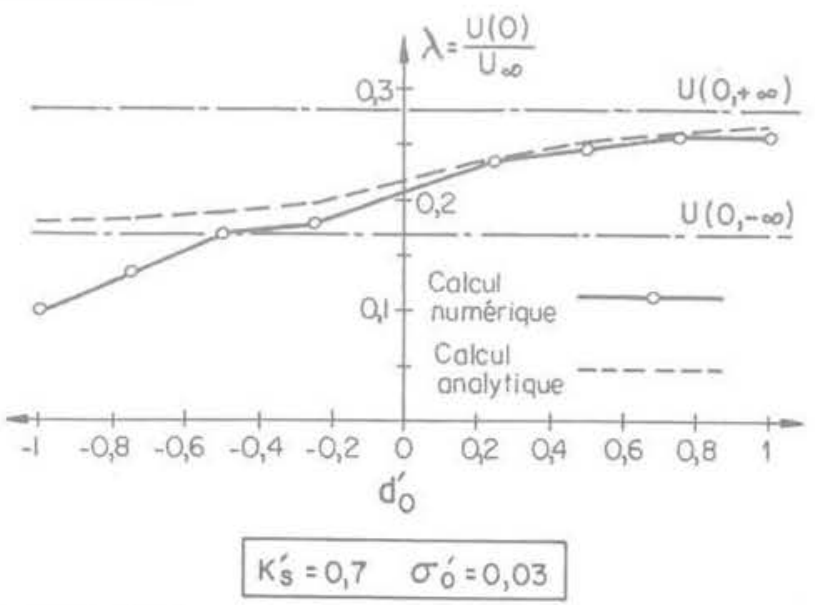

FIG. A12b

A2.

Étude la convergence en avant du front

avec:

$\mathrm{U}(\mathrm{X})=\mathrm{U}(0) \cdot \mathrm{b}(\mathrm{X})$

$b(X)=\frac{1}{1-\beta\left(v, K_{s}, d_{0}\right) \cdot x+x^{2}}$

\section{axit}

Variation de $\beta$ en fonction du coefficient de Poisson (cas du tunnel non soutenu)

$\beta(v)=\ln \left(-9 \cdot v^{2}+10 \cdot v-0,9\right)$

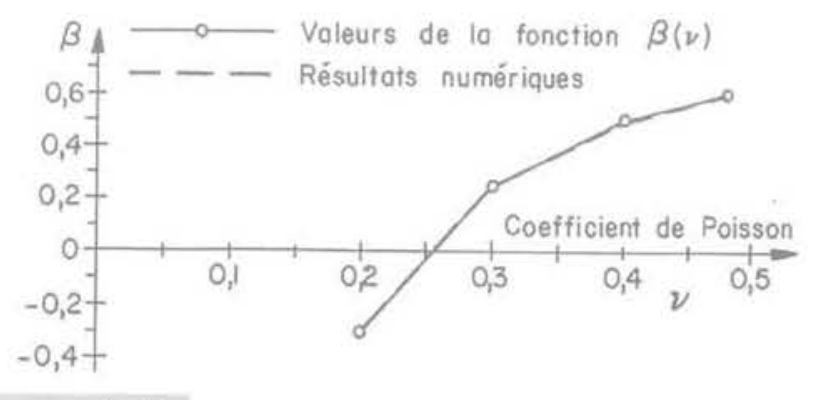

FIQ.A21

\section{A29}

Variation de $\beta$ des caractéristiques du soutènement ( $\operatorname{cas} v=0,48$ )

(Cf, tableau I au paragraphe 6.2). 


$\begin{array}{ll}K_{\mathrm{s}}^{\prime}=7 & \sigma_{0}^{\prime}=0,006 \\ d_{0}^{\prime}=-1 & \beta=-1\end{array}$

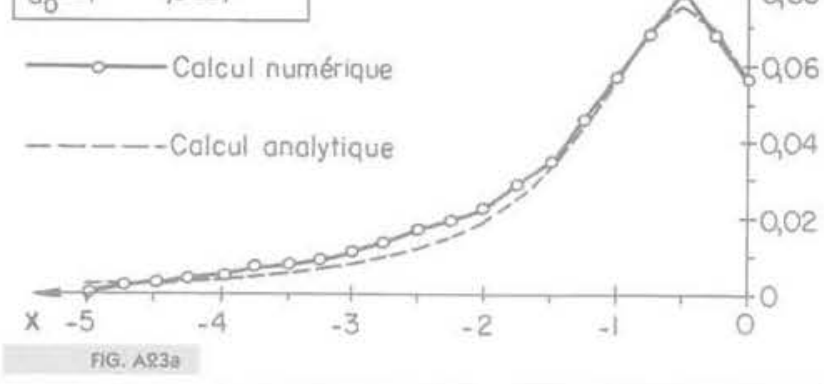

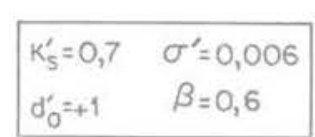
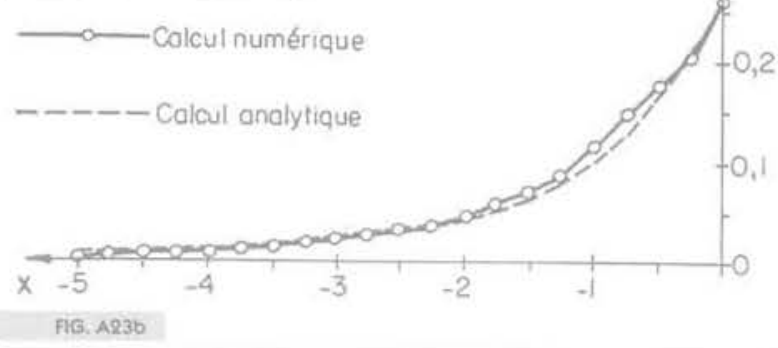

\section{Comparaison des valeurs de convergence en arrière du front pour deux cas particuliers}

\begin{tabular}{|c|c|c|c|c|c|c|c|c|c|}
\hline Cas & $d_{10}^{\prime}$ & -1 & $-3 / 4$ & $-1 / 2$ & $-1 / 4$ & $1 / 4$ & $1 / 2$ & $3 / 4$ & 1 \\
\hline \multirow{3}{*}{$\begin{array}{l}\sigma^{\prime}= \\
6.10^{-2} \\
v=0,48 \\
K_{s}^{\prime}=7\end{array}$} & $\mathrm{U}_{\mathrm{eq}}$ (numérique). $10^{-3}$ & 1.4 & 1.7 & 1,9 & 2,1 & 4,2 & 5,9 & 6,8 & 7,4 \\
\hline & $\mathrm{U}_{\mathrm{eq}}\left(\mathrm{CV}\right.$ CF/NMI) $10^{3}$ & 1,36 & 1,55 & 1,61 & 1,7 & 4,1 & 5,8 & 6,8 & 7,4 \\
\hline & $\Delta(\%)$ & 3 & 9 & 15 & 10 & 2 & 2 & 0 & 0 \\
\hline \multirow{3}{*}{$\begin{array}{l}\sigma^{\prime}= \\
3.10^{-2} \\
v=0,48 \\
K_{s}^{\prime}=0,7\end{array}$} & $U_{\text {sq }}$ (numérique). $10^{-2}$ & 24,5 & 25,0 & 27,0 & 27,5 & 35,0 & 39,0 & 40,5 & 42,0 \\
\hline & $\mathrm{U}_{x=4}(\mathrm{CVCF} / \mathrm{NMI}) 10^{-3}$ & 24,2 & 24,4 & 24,6 & 25,6 & 33,0 & 36,7 & 39,0 & 40.4 \\
\hline & $\Delta(\%)$ & 1 & 2 & 9 & 7 & 6 & 6 & 4 & 4 \\
\hline
\end{tabular}

\section{A4}

\section{Méthode de prise en compte de plusieurs type de (pré-)soutènements}

\section{ASh \\ Méthode d'étude}

On propose ici une méthode destinée à prendre en compte l'action combinée de (pré-)soutènements de

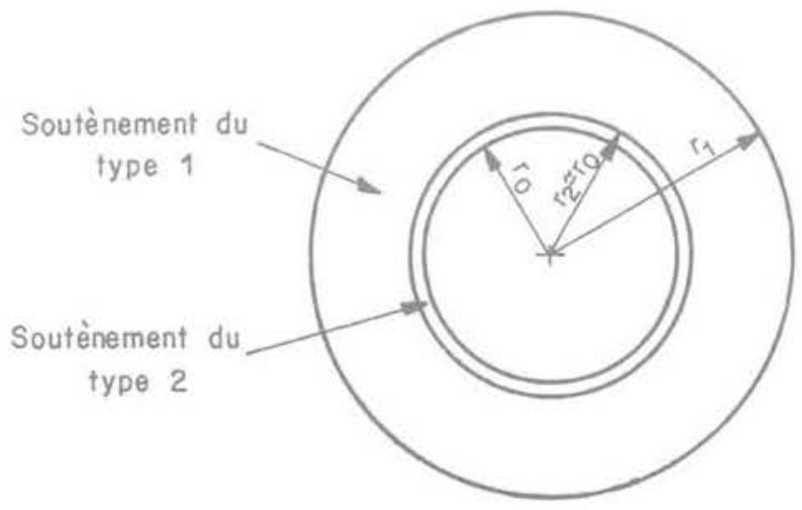

FG. A4ta Rayons d'action des différents types de soutènements. type 1 d'une part et de type 2 d'autre part. On notera $r 1$ et $r 2$ leurs rayons d'action respectifs.

- Evaluation de la convergence au niveau du front

On commencera par estimer la convergence relative $\mathrm{U}^{\prime}(\mathrm{x})$ à une distance $\mathrm{x}$ quelconque du front de taille, pour la pose uniquement des soutènements de type 1. On estimera cette convergence au niveau de la surface constituant la limite d'action de ces pré-soutènements et située à une distance $r_{1}$ de l'axe du tunnel. Cette fonction sera déterminée en considérant que les pré-soutènements de type 1 sont posés à une distance infinie du front de taille. On retiendra en particulier la valeur de la convergence au niveau du front $\mathrm{U}^{\prime}(0)$.

Le rayon de pose des soutènements de type 2 peut en revanche être assimilé au rayon d'excavation $\mathrm{r}_{\mathrm{n}}$. La convergence relative $\mathrm{U}(\infty)$ à cette distance de l'axe dans le cas de la pose des soutènements de type 1 vaut approximativement (dans le cas $v=0,5$, et considéré comme satisfaisant pour tous les cas étudiés) :

$$
U(\infty) \cdot r_{0}=\frac{r_{1}}{r_{0}} \cdot U^{\prime}(\infty) \cdot r_{1}
$$

On extrapolera ici cette relation au niveau du front en supposant que les deux courbes se déduisent l'une de l'autre par une affinité d'axe celui du tunnel et de rapport $\left(r_{1} / r_{0}\right)^{2}$. On notera $\mathrm{U}(0,+\infty)$ la valeur de $\mathrm{U}(0)$ ainsi trouvée, car elle correspond à la convergence au front dans le cas où l'on ne pose pas de soutènement de type 2. 


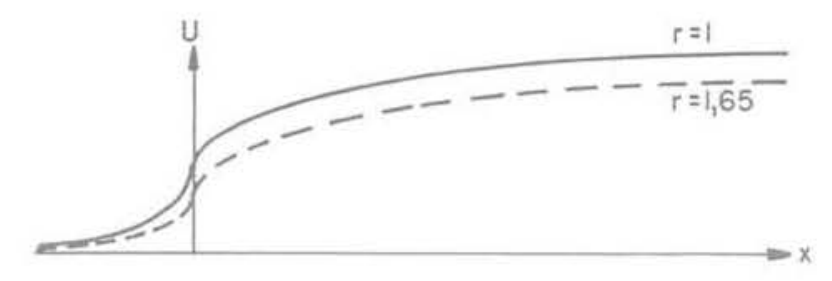

FIG A4tb Courbes de convergence du terrain à des distances différentes de l'axe du tunnel.

On notera d'autre part $\mathrm{U}(0,-\infty)$ la convergence au front dans le cas imaginaire où l'on mettrait en place les soutènements de type 2 à une distance infinie en avant du front de taille. En notant $\mathrm{K}_{3}$ ' la somme des raideurs de ces soutènements, les résultats présentés en A11 permettent d'écrire :

$$
U(0,-\infty)=\frac{U(\infty)}{(1+v) \cdot K_{2}+1} \cdot\left(0,74-\frac{1}{\left(K_{2}+3\right)^{\infty, 7}}\right)
$$

En résumé, on peut déterminer la convergence au front dans deux cas :

$-\mathrm{U}(0,+\infty)$ dans le cas d'un traitement du terrain par un pré-soutènement de type 1 (infini) et pas de soutènement de type 2 ;

$-\mathrm{U}(0,-\infty)$ dans le cas d'un traitement du terrain par un pré-soutènement de type 1 (infini) et un soutenement de type 2 infini.

La connaissance de $\mathrm{U}(0,+\infty)$ et de $\mathrm{U}(0,-\infty)$ permet de déterminer la convergence au niveau du front en tenant compte de la distance de pose et de la raideur des soutènements de type 2. Dans le cas de la pose de plusieurs soutènements successifs, on pourra considérer une distance de pose obtenu par moyenne arithmétiques des distances de pose des divers soutènements pondérés par leur raideur.

- Évaluation de la convergence en avant du front

Cette étape nécessite seulement la détermination d'une valeur du coefficient $\alpha$ tenant compte des diverses formes de (pré-)soutènements mis en place. - Évaluation de la convergence en arrière du front

Celle-ci est obtenue en appliquant une méthode adaptée au soutènement de type 2 mis en place:
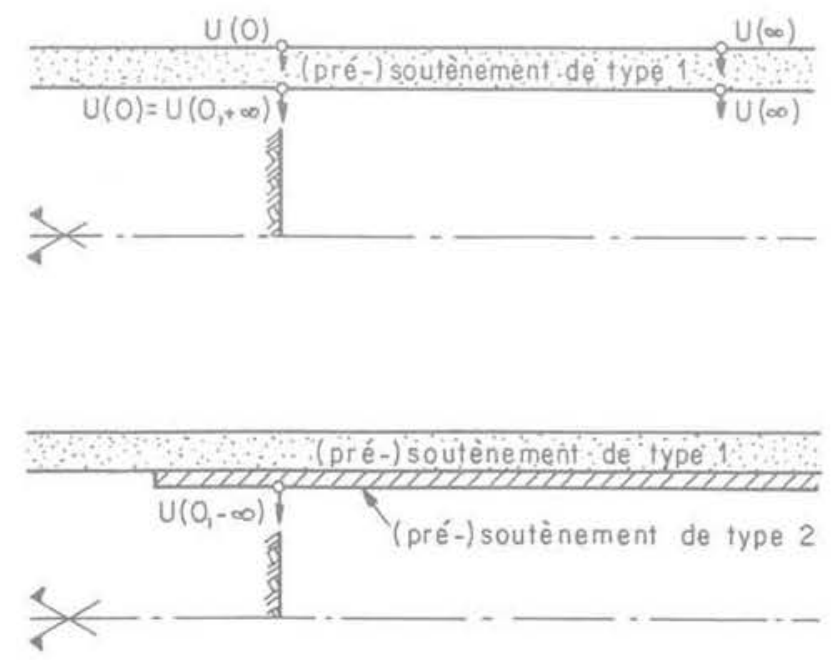

FIG. A41c Notations utilisées. méthode convergence-confinement s'il s'agit de présoutènement, Nouvelle Méthode implicite s'il s'agit de soutènement classique.

\section{AS9 \\ Exemple}

Mise en place d'une voûte-parapluie (rayon d'excavation $=4 \mathrm{~m}$, longueur des barres $=16 \mathrm{~m}$, recouvrement $=8 \mathrm{~m}$ ).

Paramètres adimensionnels : $v=0,48 ; \sigma_{0}^{\prime}=0,006$; $\mathrm{K}_{1}^{\prime}=4.72 ; \mathrm{K}_{2}^{\prime}=7 ; \mathrm{d}_{0}^{\prime}=1 / 4$

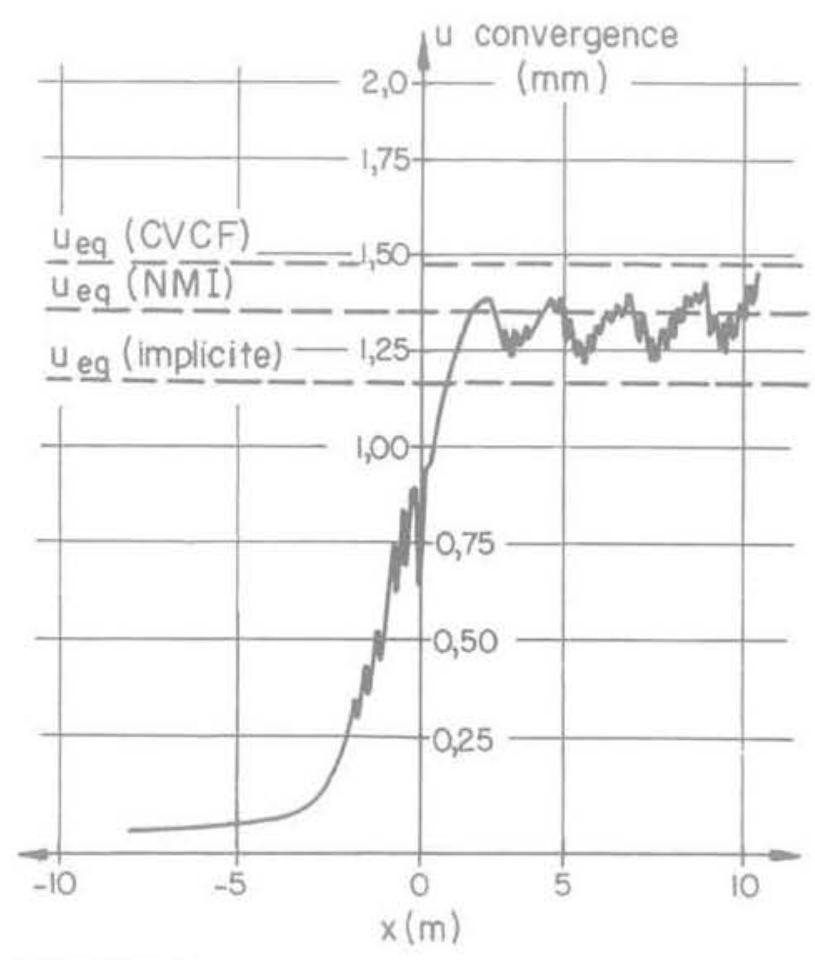

HG: $A 42$

\section{Annexe B}

\section{Résultats obtenus en plasticité (cas Ns = 2)}

Les analyses sur la convergence en avant du front montrent que l'on peut utiliser le même type de relation entre $\mathrm{U}(\mathrm{X})$ et $\mathrm{U}(0)$ qu'en élasticité, avec une fonction $\beta$ dépendant :

- très peu du coefficient de Poisson v (Fig. B1) ;

- très peu de la distance de pose d'。(Fig. B2) ;

- assez nettement de la raideur du soutènement $K^{\prime}$, (Fig. B3), notamment pour les fortes raideurs : pour $\mathrm{K}_{\text {s }}^{\text {s }}$ $<0,1$, on peut retenir $\beta=1,3$; au-delà on retiendra une loi de variation telle que représentée sur la figure 9 dans le texte. 

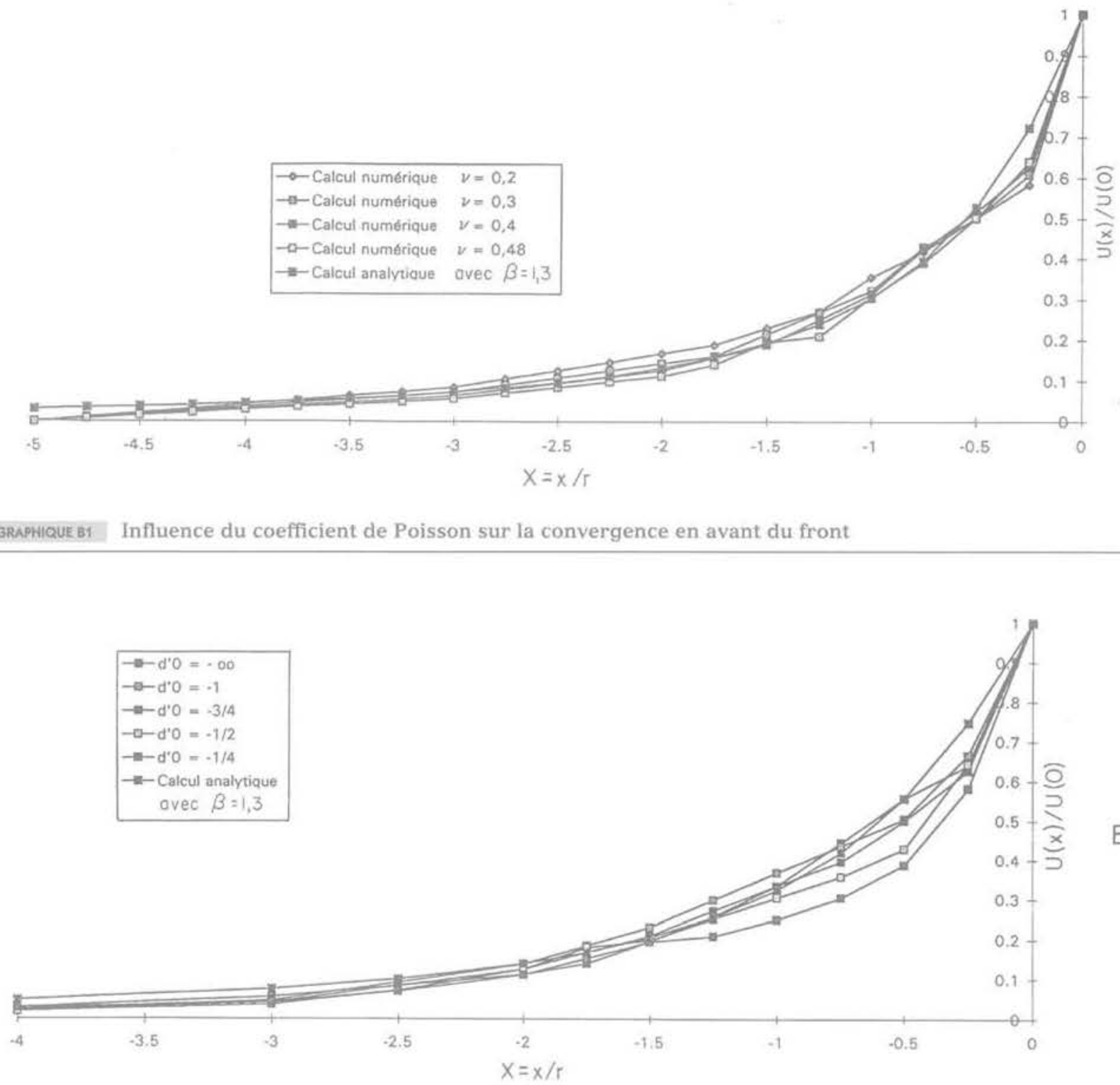

B2

GRAPHIQUE: Influence de la distance de pose sur la convergence en avant du front.

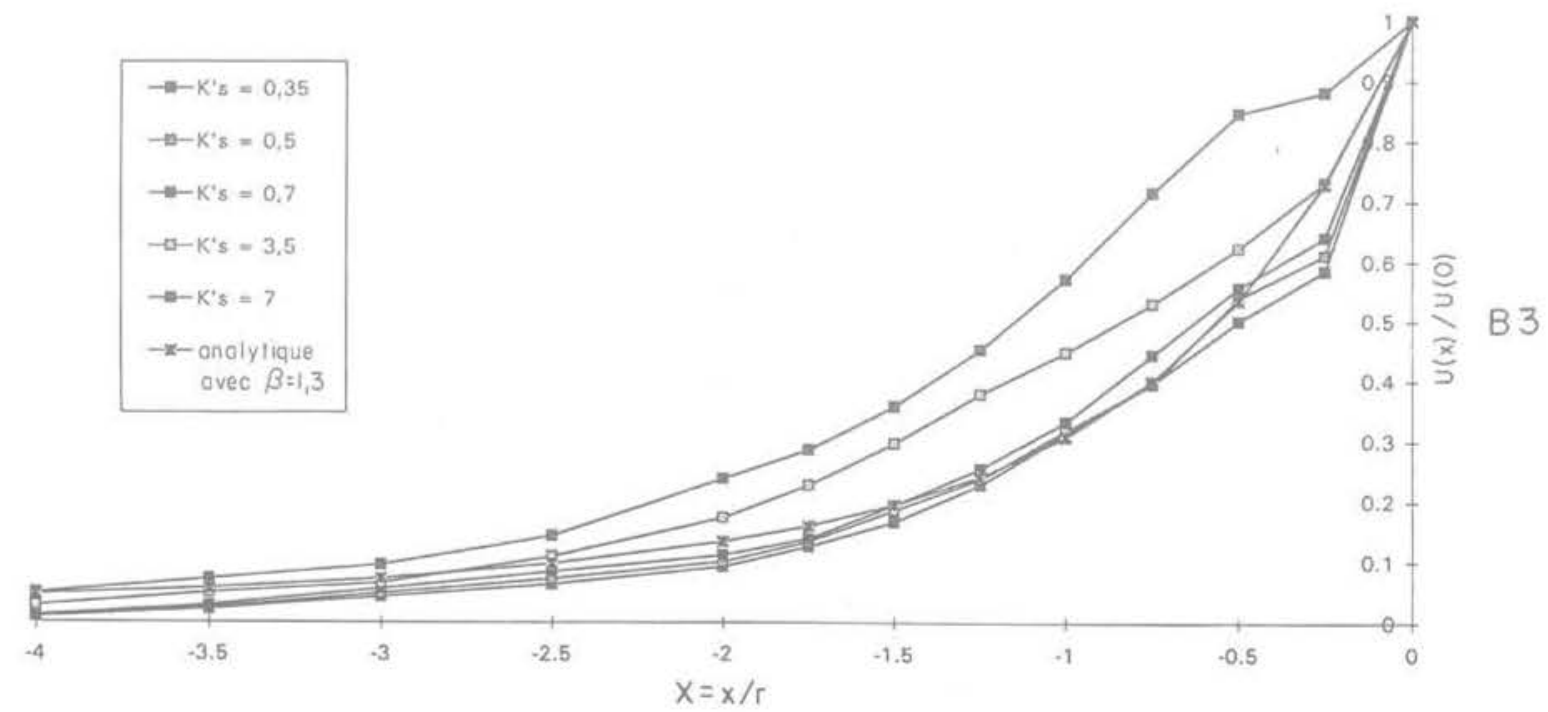

GRAPHIQUE B3 Influence de la raideur du soutènement sur la convergence en avant du front. 